Analytical Letters, Volume 42, Issue 17, January 2009, pages 2905-2913

\title{
A label free electrochemical nanobiosensor study
}

Juankun Zhang ${ }^{1}$, Shanmu Dong ${ }^{1}$, Jinhui Lu ${ }^{1}$, Anthony P F Turner ${ }^{2}$, Qingjie Fan ${ }^{3}$,

Shiru Jia ${ }^{1}$, Hongjiang Yang ${ }^{1}$, Changsheng Qiao ${ }^{1}$, Hao Zhou ${ }^{1}$ and Guowei $\mathrm{He}^{4}$

${ }^{1}$ Key Laboratory of Industrial Microbiology, Ministry of Education, College of Biotechnology, Tianjin University of Science and Technology, Tianjin, P. R. China

${ }^{2}$ Cranfield Health, Cranfield University, Cranfield, Bedfordshire MK43 0AL, UK.

${ }^{3}$ Lanlike Electron Chemistry High-Tech Ltd., Tianjin, P. R. China

${ }^{4}$ TEDA International Cardiovascular Hospital, No.61, Third Avenue, TEDA, Tianjin, P, R. China

* Corresponding author. Tel.: 00862260600063 , Fax: 00862260602298

E-mail address: zhangjk @ tust.edu.cn 


\begin{abstract}
Nano-porous silicon (PS) is an attractive material for incorporation into biosensors, because it has a large surface area combined with the ability to generate both optical and electrical signals. In this paper, we describe a label-free nanobiosensor for bovine serum albumin (BSA). Nano-porous silicon produced in our laboratory was functionalised prior to immobilisation of anti-BSA antibody on the surface. Reaction with BSA in phosphate buffered saline (PBS) buffer resulted in an impedance change which was inversely proportional to the concentration of the analyte. The system PBS buffer/antigen-antibody/PS constitutes an electrolyte-insulator-semiconductor (EIS) structure, thus furnishing an impedance EIS nanobiosensor. The linear range of the sensor was $0-0.27 \mathrm{mg} \mathrm{mL}^{-1}$ and the sensitivity was less than $10 \mu \mathrm{g} \mathrm{mL}^{-1}$.
\end{abstract}

Keyword : nano-porous silicon; nanobiosensor; electrolyte-insulator-semiconductor; impedance 


\section{Introduction}

Nano-porous silicon (PS) has received considerable recent attention for incorporation into biosensors due to its intrinsic optic and electrical properties and its large surface area, which can be readily functionalised (Zhang et al. 2007). Lin et al. (Lin et al. 1997) reported an optical interferometric biosensor based on porous silicon, which was capable of detecting a wide range of analytes with low detection limits. A capacitive catalytic biosensor has also been described with sensitivity for penicillin of around $0.25 \mathrm{mM}$ (Thust et al. 1999). The authors highlighted the large amount of immobilised enzyme associated with the porous structures. Chan and co-workers designed a DNA sensor using a Bragg reflector. When a biological molecule attached to the large internal surface of the porous silicon, a change in refractive index was detected by the shift in the luminescence peaks (Chan et al. 2001). In 2007, we reported a simple, label-free optical sensor for BSA. The detection principle was based on the change in light intensity associated with the interaction of protein molecules with the nano-PS surface (Zhang et al. 2007).

Sensors for biological analytes based on electrolyte-insulator-semiconductor (EIS) structures have been reported since the late 1990s (Brett et al. 2001; Berney et al. 1999; Schoning et al. 2002; Bergveld et al. 2003; Schoning et al. 2005; Poghossian et al. 2006). The EIS structure is similar to the metal-insulator- semiconductor (MIS), but the metal contact on the insulator is replaced by an electrolyte contact, to which a voltage is applied by means of a reference electrode. However, very few articles have addressed EIS biosensors using PS. Porous EIS structures of $n-\mathrm{Si} / \mathrm{SiO}_{2} / \mathrm{Si}_{3} \mathrm{~N}_{4}$ have 
been realised for capacitive $\mathrm{pH}$ sensors. The average $\mathrm{pH}$ sensitivity of the porous $\mathrm{pH}$ sensor amounts to about $56 \mathrm{mV} / \mathrm{pH}$ in the range between $\mathrm{pH} 4$ and $\mathrm{pH} 8$. The study also utilised penicillinase as a model enzyme to prepare a porous EIS biosensor and the sensitivity reported was around $0.25 \mathrm{mM}$ (Schoning et al. 2000). Betty and co-workers (Betty et al. 2004; Betty et al. 2007) described the fabrication and characterisation of capacitive biosensors with EIS structures and conducted a time response study. They found that there was a rapid decrease in capacitance following addition of the analyte to the PS sensor.

In the present work, we describe an impedance immunosensor based on porous EIS technology comprising a PBS buffer/antibody-antigen/PS structure. The specific reaction of the antigen (BSA) to the immobilized antibody (antiserum of BSA) caused a change in impedance that could be monitored on-line. The sensitivity of the electro-PS biosensor was also investigated.

\section{Experimental}

\subsection{PS preparation}

Nano-porous silicon was obtained from p-type $\operatorname{Si}(100)$ with a specific resistance $\rho=0.15-0.2 \Omega . \mathrm{cm}$, using an electrochemical etching process at $220 \mathrm{~mA} / \mathrm{cm}^{2}$ for 20 minutes in $48 \%$ hydrofluoric acid (HF) solution. The PS chip was then treated with $\mathrm{H}_{2} \mathrm{O}_{2}$ to oxidize the PS surface (Zhang et al. 2007). 


\subsection{Antibody immobilisation}

The Anti-BSA antibody (from Dingguo company) immobilisation was carried out by immersing $0.5 \mathrm{~cm}^{2}$ PS chips into antibody solution for one hour at room temperature. After thoroughly washing with PBS, the nonspecific sites were blocked with $2 \mathrm{mg} / \mathrm{ml}$ Ovalbumin, then washed again with PBS thoroughly.

The antibody immobilisation on the porous silicon matrix was confirmed by JSPM-5200 atomic force microscopy from JEOL, Japan.

The activity of the immobilised antibody was visualized using BSA - colloidal gold .

The gold nanoparticles were produced by dissolving $\mathrm{H}\left[\mathrm{AuCl}_{4}\right]$, the solution was rapidly stirred while a reducing agent was added. This caused $\mathrm{Au}^{3+}$ ions to be reduced to neutral gold atoms, and the gold gradually starts to precipitate in the form of sub-nanometer particles. If the solution is stirred vigorously enough, the particles will be fairly uniform in size.

The antigen colloidal gold labeling was taken by the electrostatic interaction between Au clusters and the protein amino group. The BSA- colloidal gold was prepared by mixing colloidal gold suspension with 0.1M BSA solution. After separation, the BSA-colloidal gold was applied on the antibody-PS and on the free PS without antibody, but where the protein binding sites were blocked with Ovalbumin. If the immobilised antibody was active, the BSA - colloidal gold bound to the antibody-PS and presented a dark red color. In contrast, the colour of the free PS was light. 


\subsection{BSA determination using the impedance method}

The impedance analysis was performed using an LK2005A electrochemical work station (supplied by Lanlike Co Ltd, Tianjin, China). The system consisted of an $\mathrm{Ag} / \mathrm{AgCl}$ reference electrode, the antibody-immobilised PS chip as the working electrode and a platinum wire counter electrode. The buffer was 0.015M PBS, pH 7.4. The impedance was measured with the substrate bias voltage range of $0.15 \mathrm{~V}$ to $0.29 \mathrm{~V}$. The analysis commenced with $10 \mathrm{ml}$ of $0.015 \mathrm{M}$ PBS buffer and then serial concentrations of BSA (0.01, 0.02, 0.04, 0.08, 0.1 mg/ml) were added into the system. The impedance versus BSA concentration was plotted.

The selectivity of the antibody-PS was also identified with ovalbumin (OVA). At the same condition, the same concentration of ovalbumin (OVA) and the BSA were added into the system to react with the antibody-PS, and then the changes of impedance were collected.

\section{Results and Discussion}

\subsection{Confirmation of the presence of immobilised antibody}

In order to confirm the antibody was immobilised on the PS chip the antibody-immobilised PS and the free PS chips were scanned with a atomic force microscopy (figure 1). It shows that the antibody immobilised PS chip (figure 1b) is more than triple thicker than the free chip (figure 1a). This reveals that the antibody has been immobilised on the porous silicon matrix. 
The activity of the antibody on the chip was determined by reacting the chips with BSA-colloidal gold. As shown in figure 2, fig $2 \mathrm{a}$ is darker than fig $2 \mathrm{~b}$. This reveals that the BSA-colloidal gold had bound to the antibody-immobilised PS chip and the antibody activity was retained.

\subsection{Comparison of impedance changes with antibody- immobilised and free PS}

Plots of the impedance versus voltage, before and after the deposition of the antibody layer, are presented in Figure 3 a. The deposition of the antibody layer led to a decrease in impedance. The decrease in impedance is thought to be due to a change in dielectric constant after antibodies were immobilised on the PS surface (Figure $3 \mathrm{~b}$ ). The electrolyte used for the measurements was 0.015M PBS.

\subsection{BSA determination}

The selectivity of the antibody-PS was checked with OVA. When the BSA-antibody-PS reacted with the same concentration of BSA and OVA, the reduction of impedance with BSA is about $60 \%$ larger than with OVA (figure 4). Therefore, the biosensor has a significant and specific selectivity.

Figure 5 shows the impedance versus voltage plots of the PS sensors after equilibration with different BSA concentrations for 1 minute, and the total impedance is decreased after adding BSA.

By fixing the applied potential during in the accumulation of protein at $0.29 \mathrm{~V}$, the impedance varied inversely with the concentration of the analyte, BSA (Figure 6). 
The detection limit is $10 \mathrm{mg} / \mathrm{ml}$. it equals 3 _ SD of zero calibrator. Furthermore, Figure 6 illustrates that the linear range of the sensor is between $0-0.27 \mathrm{mg} / \mathrm{ml}$.

The material used in the literatures is mainly plain silicon. Our material is Nano-porous silicon, it has many advantages, such as it possess of a large surface area with optical and electrical signals. The novelty of the work is to combine the nano-porous silicon properties with the biosensor technology to produce a label free nano-biosensor with a linear range of $0.01-0.08 \mathrm{mg} / \mathrm{ml}$. The sensitivity of the biosensor was $10 \mu \mathrm{g} / \mathrm{ml}$.

\section{Conclusion}

Nano-porous silicon multilayered microstructures have unique optical, electronic and morphological properties that can be exploited in chemical and biological sensing. The large specific surface of nano-structured porous silicon can be chemically modified to link different molecular probes, which recognise the target analytes. In order to enhance the sensitivity and simplicity of this as a sensor device, we have produced and characterised several porous silicon-based structures (Zhang et al. 2007). In this paper, we developed a label free nanobiosensor for bovine serum albumin (BSA) determination. Using functionalized PS with immobilised anti-BSA antibody we showed that the impedance decreased on binding the complimentary antigen and that the linear range of the sensor was between $0-0.27 \mathrm{mg} / \mathrm{ml}$. The minimum detectable amount of antigen was less than $10 \mu \mathrm{g} / \mathrm{ml}$. 


\section{Acknowledgements}

The authors would like to thanks the Ministry of Education, the Tianjin MunicipalScience and Technology Commission (09ZCZDSF04200 and 09ZXCXGX17100), and Tianjin University of Science and Technology for supporting this work.

\section{References}

Bergveld, P. 2003. Thirty years of ISFETOLOGY - What happened in the past 30 years and what may happen in the next 30 years. Sens. Actuators B. 88: 1 .

Berney, H., West, J., Haefele, E., Alderman, J., Lane, W., Collins, J.K. 1999. A DNA diagnostic biosensor: development, characterisation and performance. Sens. Actuators B. $68: 100$.

Betty, C.A., Lal, R., Sharma, D.K., Yakhmi, J.V., Mittal, J.P., 2004. Macro- porous silicon based capacitive affinity sensor-fabrication and electrochemical studies. Sens. Actuators B. 97: 334.

Betty C.A., Lal, R., Yakhmi, J.V., Kulshreshtha, S.K. 2007. Time response and stability of porous silicon capacitive immunosensors. Bioisens. Bioelectron. 22: 1027.

Brett, C.M.A., Brett, A.M.O., Serrano, S.H.P. 2001. An EIS study of DNA- modified electrodes. Electrochim. Acta. $44: 4233$.

Chan, S., Li, C.S., Lewis, Y., Rothberg, J., Miller, B.L., Fauchet, P.M. Mat. 2001. Nanoscale silicon microcavities for biosensing. Sci. Eng. C-Bio. 15: 277. 
Lin, V.S., Motesharie, K., Dancil, K.P.S., Sailor, M.J., RezGhadiri, M. 1997. A porous silicon based optical interferometric biosensor. Science. 278: 840.

Poghossian, A., Schoning, M.J. 2006. Silicon-based chemical and biological field-effect sensors. Encyclopedia of Sensors.9: 463.

Schoning, M.J., Poghossian, A. 2002. Recent advances in. biologically sensitive field-effect transistors (BioFETs). Analyst.127:1137.

Schoning, M.J. 2005. "Playing around" with field-effect sensors on the basis of EIS s tructures, LAPS and ISFETs. Sensors. 5: 126.

Schoning, M.J., Kurowski, A., Thust, M., Kordos, P., Schultze, J.W., Luth, H. 2000. Capacitive microsensors for biochemical sensing based on porous silicon technology. Sens. Actuators B. 64: 59.

Thust, M., Schoning, M.J., Schroth, P., Malkoc, U., Dicker, C.I., Steffen, A., Kordos, P., Luth, H. 1999. Enzyme immobilisation on planar and porous silicon substrates for biosensor applications. J. Mol. Catal. B: Enzym. 7:77.

Zhang, J., Zhang, W., Dong, S., Turner, A.P.F., Fan, Q., Jia S. 2007. Light- Emitting Silicon Chip as a Potential Biosensor Platform. Anal. Lett. 40: 1549. 


\section{Legends}

Figure 1. The atomic force microscopy image of free PS (A) and antibody immobilised PS (B).

Figure 2. To identify the presence of the immobilised anti-BSA antibody, the chips were treated with BSA-colloidal gold: (A) the antibody-PS chip, (B) the free PS chip Figure 3. (A) shows the impedance versus voltage plots before and after the deposition of the antibody layer in $0.015 \mathrm{M}$ PBS, pH 7.4. Insert shows the impedance reduction after antibody immobilization in the voltage range of $0.15 \mathrm{~V}$ to $0.29 \mathrm{~V}$ (B) describes the principle of the impedance decreases after antibody is bound to the PS electrode.

Figure 4. The selectivity of the antibody-PS was checked with reacting to the same concentration of BSA and OVA, The reduction of impedance with BSA is larger than with OVA.

Figure 5. The impedance versus voltage plots of the porous silicon sensors after addition of different BSA concentrations, 0.01, 0.03, 0.13, 0.23 and $0.27 \mathrm{mg} / \mathrm{ml}$, respectively.

Figure 6. The effect of BSA concentration on impedance at $290 \mathrm{mV}$ from figure 5. 
Figures

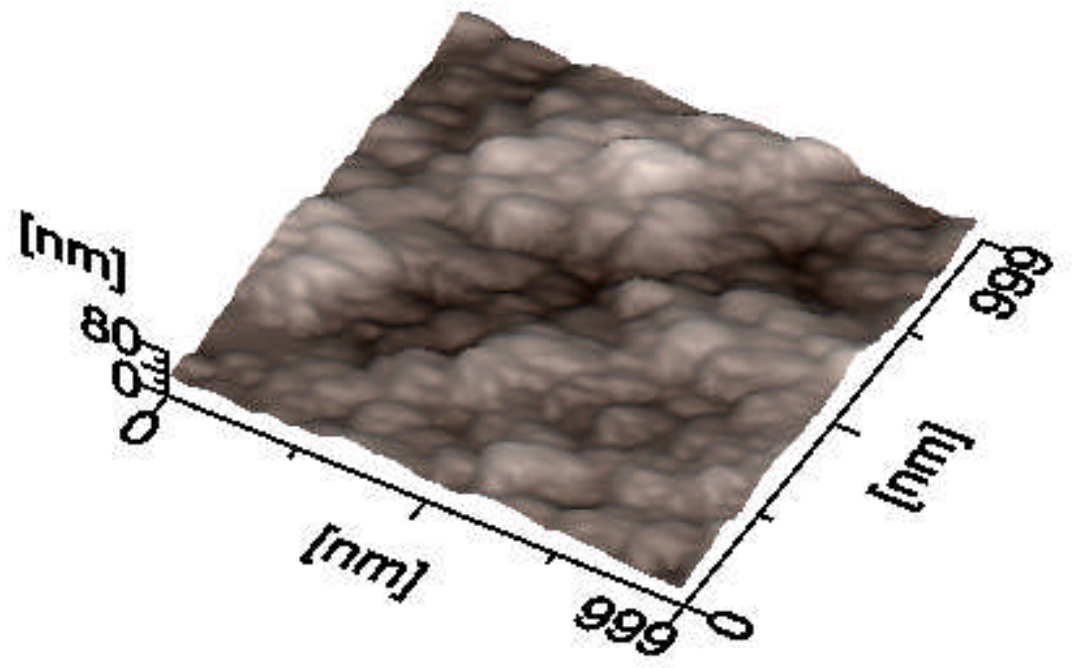

(a)

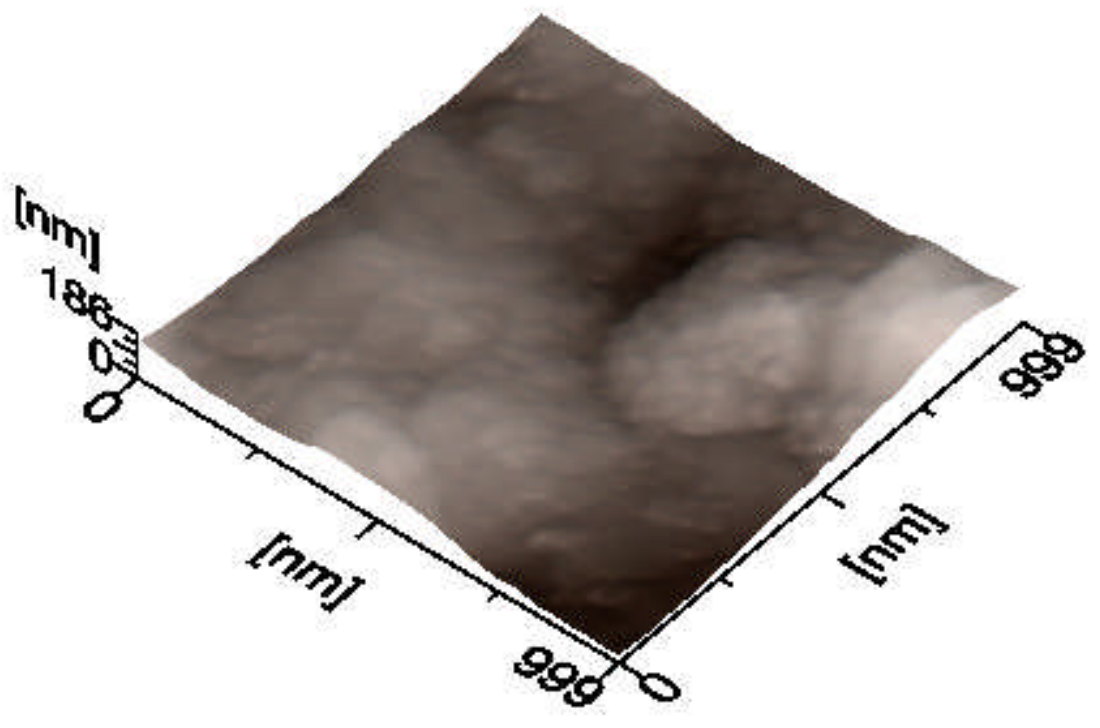

(b)

Figure 1 


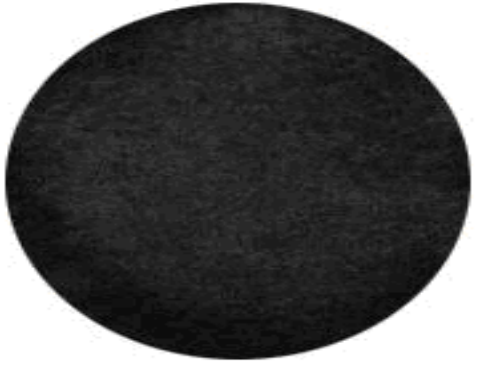

(a)

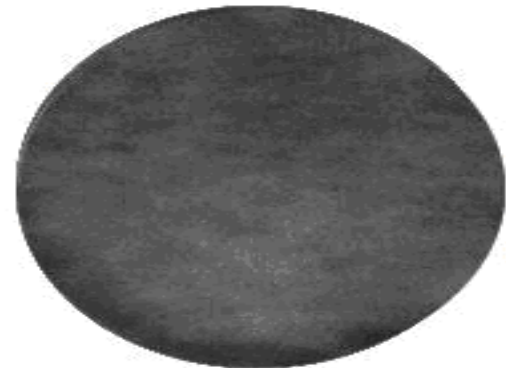

(b)

Figure 2 

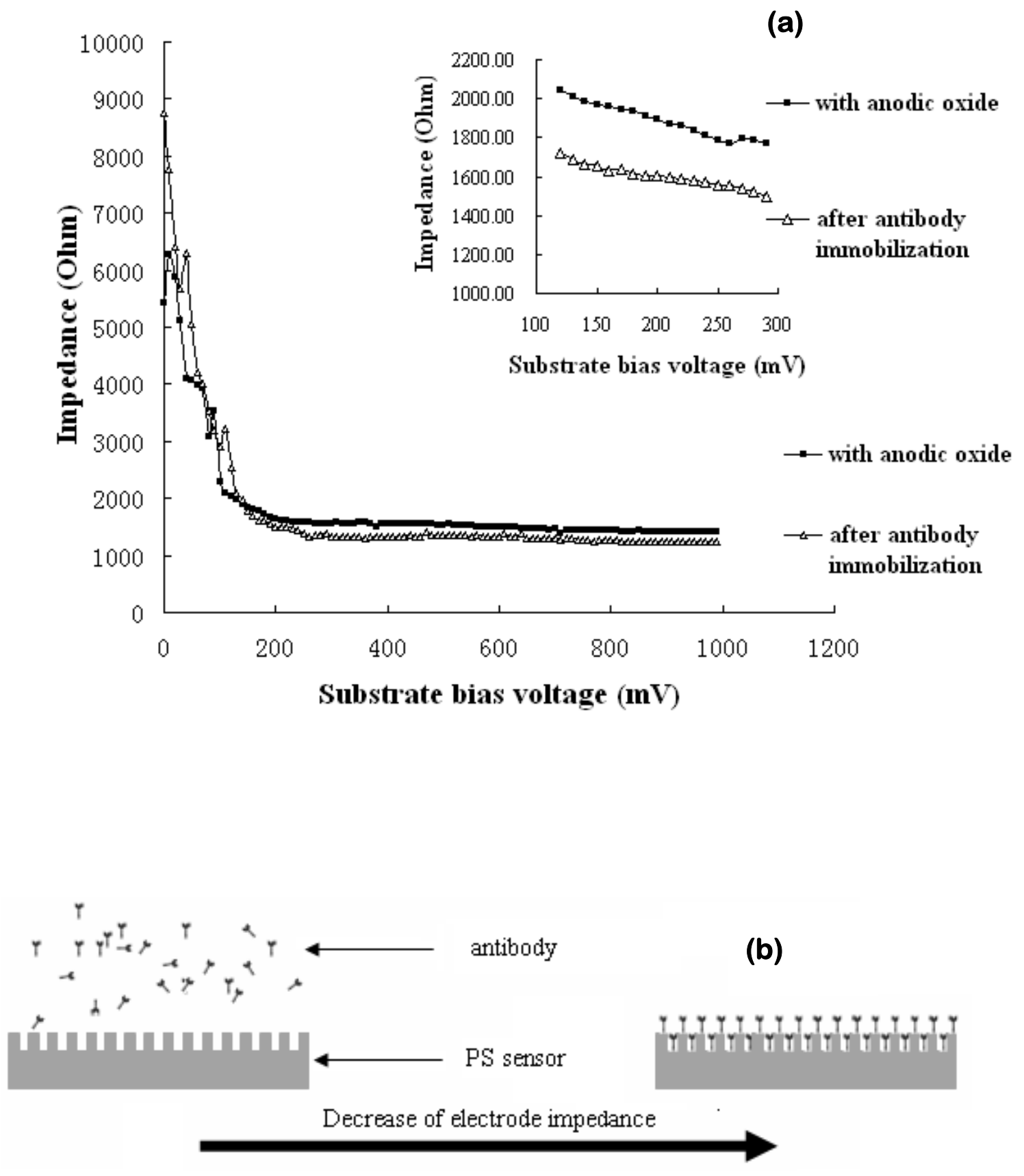

Figure 3 


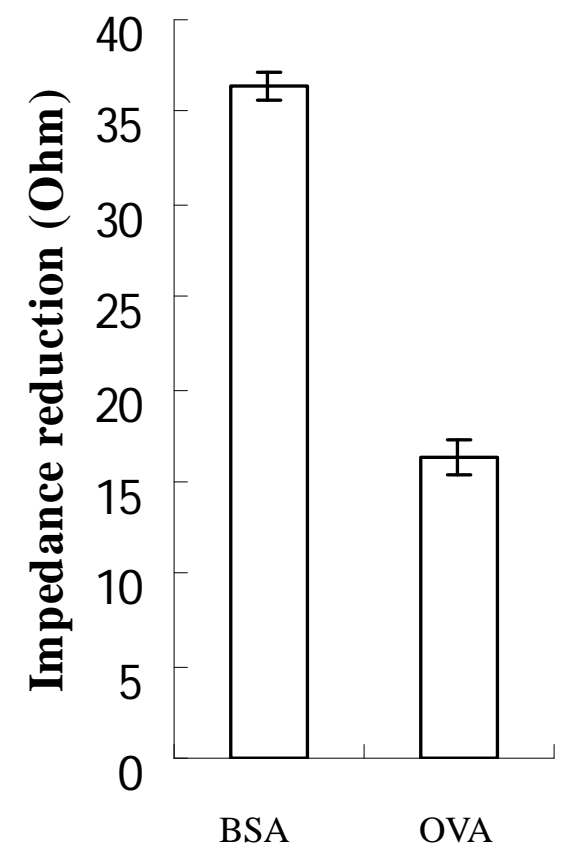

Figure 4 


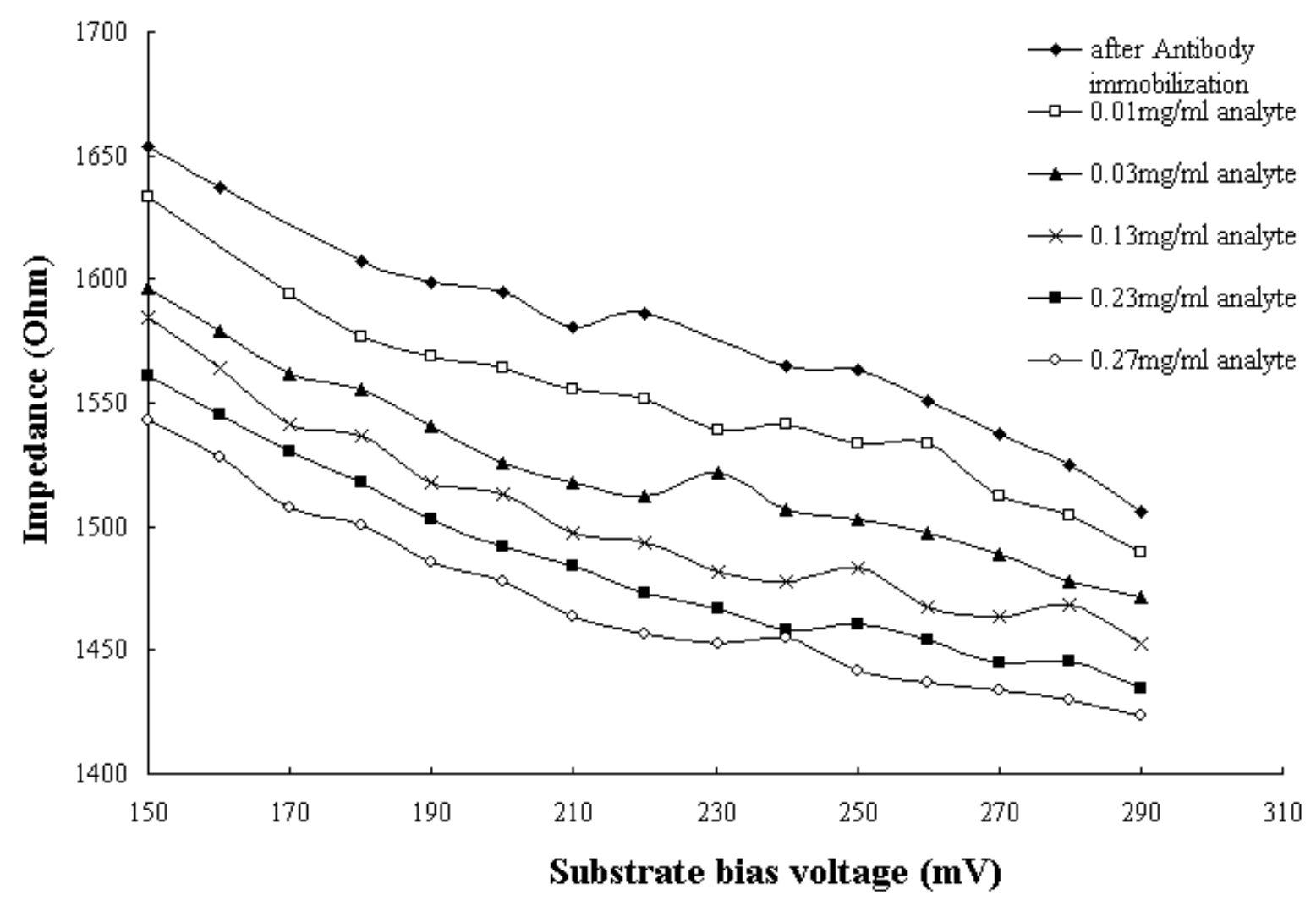

Figure 5 


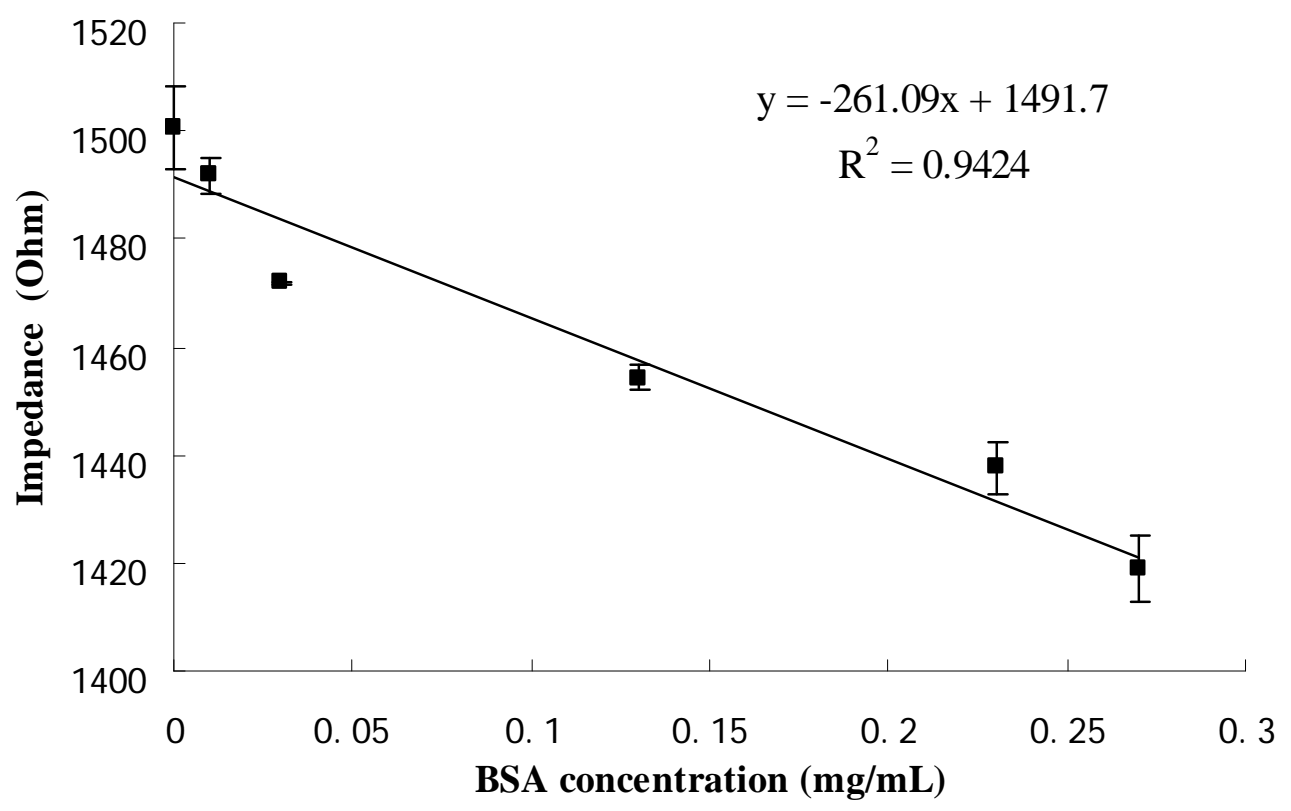

Figure 6 